\title{
Generalized statistical mechanics of cosmic rays
}

\author{
Christian Beck
}

\author{
School of Mathematical Sciences, Queen Mary, University of London, Mile \\ End Road, London E1 4NS, UK.
}

\begin{abstract}
We consider a generalized statistical mechanics model for the creation process of cosmic rays which takes into account local temperature fluctuations. This model yields Tsallis statistics for the cosmic ray spectrum. It predicts an entropic index $q$ given by $q=11 / 9$ at largest energies (equivalent to a spectral index of $\alpha=5 / 2$ ), and an effective temperature given by $\frac{5}{9} T_{H}$, where $k T_{H} \approx 180 \mathrm{MeV}$ is the Hagedorn temperature measured in collider experiments. Our theoretically obtained formula is in very good agreement with the experimentally measured energy spectrum of primary cosmic rays.
\end{abstract}


More general versions of statistical mechanics, as introduced by Tsallis [1] and further developed by many others 2, 3, have recently been successfully applied to a variety of complex physical systems. The idea is to maximize more general entropy measures than the Shannon entropy, which depend on a parameter $q$ and which lead to generalized versions of statistical mechanics. Ordinary statistical mechanics is contained as a special case for $q=1$. Interesting recent physical applications of the so-called nonextensive formalism obtained for $q \neq 1$ include the statistics of fully developed hydrodynamic turbulence 4], defect turbulence [5], scattering processes in $e^{+} e^{-}$annihilation [6. 7], heavy ion collisions [8], hadron collisions [9], and models of vacuum fluctuations at the Planck scale [10]. There is growing evidence that the nonextensive formalism, though possessing the mathematical structure of an equilibrium formalism, is physically often relevant for nonequilibrium systems with a stationary state that possess strong fluctuations of an intensive parameter [11, 12, 13.

Very recently Tsallis and Borges [14] analysed the experimentally measured energy spectrum of cosmic rays from a nonextensive point of view. They showed that a good fit of the measured spectrum can be obtained by assuming the interplay of two different nonextensive canonical distributions with two different entropic indices $q_{1}$ and $q_{2}$ and two different inverse temperatures $\beta_{1}$ and $\beta_{2}$. Moreover, Kaniadakis [15] showed that yet another type of generalized statistical mechanics (based on entropies that are neither Shannon nor Tsallis) also yields a good fit of the measured spectrum, using three fitting parameters. Both these papers represent new interesting ideas, but at the same time they use various fitting parameters which are not predicted from first principles. The question arises whether one can construct a generalized statistical mechanics model of cosmic rays that predicts the relevant parameters from first principles and that at the same time well reproduces the measured cosmic ray energy spectrum using these parameters.

In this letter we consider such a generalized statistical mechanics model. The model is quite generally of relevance for particles that are created by scattering processes at very large energies (as cosmic ray particles are). The basic idea is that at very high energies the effective thermodynamic interaction volume is small and hence one expects that there are strong local temperature fluctuations. A relevant thermodynamic theory of high-energy scattering processes is the Hagedorn theory [16], which we will generalize here in the sense that we take into account local temperature fluctuations. Under reasonable assumptions on the form of the temperature fluctuations 
this model predicts Tsallis statistics for the cosmic ray spectrum. More importantly, it yields a concrete prediction of the entropic index $q$ (or the differential spectral index $\alpha$ of the cosmic flux) and of the relevant effective temperature that enters into the generalized canonical distributions. Our model turns out to yield a very good fit of the experimentally measured cosmic ray spectrum. The advantage of our model is that the most relevant parameters are calculated, not fitted. The more subtle features of the cosmic ray spectrum (such as the well-known 'knee' and 'ankle') are then finally related to the existence of two different populations of cosmic rays, each being described by the above nonextensive theory (with the same parameters) but having a different flux rate.

To start with, let us first have a look at the experimental data as collected from various experimental groups [17. These data are displayed in Fig. 1. Also shown is a curve that corresponds to a prediction of nonextensive statistical mechanics. Up to energies of $10^{16} \mathrm{eV}$, the measured flux rate of cosmic ray particles with a given energy is well fitted by a generalized canonical distribution of the form

$$
p(E)=C \cdot \frac{E^{2}}{(1+\tilde{\beta}(q-1) E)^{1 /(q-1)}} .
$$

Here $E$ is the energy of the particles

$$
E=\sqrt{c^{2} p_{x}^{2}+c^{2} p_{y}^{2}+c^{2} p_{z}^{2}+m^{2} c^{4}},
$$

$\tilde{\beta}=(k \tilde{T})^{-1}$ is an effective inverse temperature variable, and $C$ is a constant representing the total flux rate. For relativistic particles the rest mass $m$ can be neglected and one has

$$
E \approx c|\vec{p}|
$$

The distribution (11) is a $q$-generalized relativistic Maxwell-Boltzmann distribution in the formalism of nonextensive statistical mechanics. These kind of distributions can be directly obtained by maximizing the Tsallis entropies [1]

$$
S_{q}=\frac{1}{q-1}\left(1-\sum_{i} p_{i}^{q}\right)
$$

and multiplying with the available phase space volume [6, 7, 14]. The $p_{i}$ are the probabilities of the microstates $i$. As seen in Fig. 1, the cosmic ray 
spectrum is very well fitted by the distribution (II) if the entropic index is chosen as

$$
q=1.215
$$

and if the effective temperature parameter is given by

$$
k \tilde{T}=\tilde{\beta}^{-1}=107 \mathrm{MeV} .
$$

Our main aim in the following is to derive the form of the distribution (11) from physically reasonable assumptions, based on ordinary statistical mechanics but leading effectively to a nonextensive description. Moreover, we will derive concrete numerical values for the two parameters $q$ and $\tilde{\beta}$, thus proceeding from a mere fit towards a theory.

Cosmic ray particles are created in high energy collision processes as induced by strong galactic and extragalactic 'accelerators' (see e.g. 18] for a review). These 'accelerators' are astrophysical sources and may produce a center of mass energy $E_{C M S}$ of the collision process in the $\mathrm{GeV}$ and $\mathrm{TeV}$ region, but much larger energies are possible as well. In general, from a statistical mechanics point of view it is well known that the largest average temperature that can be reached in high energy collision processes is the Hagedorn temperature $T_{H}$ [16], experimentally measured to be about $180 \pm 30 \mathrm{MeV}$ [19, 20]. The Hagedorn temperature is much smaller than $E_{C M S}$ and represents a kind of 'boiling temperature' of nuclear matter at the confinement phase transition. Even largest $E_{C M S}$ cannot produce a larger average temperature than $T_{H}$ due to the fact that the number of possible particle states grows exponentially.

Let us now provide a plausible physical argument how nonextensive behavior comes into play at very large energies. This argument is just based on ordinary statistical mechanics but takes into account fluctuations. The larger the center of mass energy of the collision process, the smaller the volume probed, due to the uncertainty relation. This means, the effective interaction volume where a thermodynamic description of the collision process makes sense will become smaller and smaller with increasing $E_{C M S}$. However, a smaller volume means larger temperature fluctuations. It thus makes sense to consider a generalized Hagedorn theory which takes into account local temperature fluctuations.

Assume that locally some value of the fluctuating inverse temperature $\beta$ is given. We then expect the momentum of a randomly picked particle in this region to be distributed according to the relativistic Maxwell-Boltzmann 
distribution

$$
p(E \mid \beta)=\frac{1}{Z(\beta)} E^{2} e^{-\beta E} .
$$

Here $p(E \mid \beta)$ denotes the conditional probability of $E$ given some value of $\beta$. We neglect the rest mass $m$ so that $E=c|\vec{p}|$. The normalization constant is given by

$$
Z(\beta)=\int_{0}^{\infty} E^{2} e^{-\beta E} d E=\frac{2}{\beta^{3}} .
$$

Now let us take into account local temperature fluctuations in the small interaction volumes where cosmic ray particles are produced. We have to consider some suitable probability density $f(\beta)$ of the inverse temperature in the various interaction volumes. While the most natural probability distribution of a continuous random variable taking positive and negative values is the Gaussian distribution, the most natural distribution of a continuous random variable living on a positive support ( $\operatorname{such}$ as $\beta$ ) is the $\chi^{2}$-distribution of degree $n$, i.e. the probability density of $\beta$ is given by

$$
f(\beta)=\frac{1}{\Gamma\left(\frac{n}{2}\right)}\left\{\frac{n}{2 \beta_{0}}\right\}^{\frac{n}{2}} \beta^{\frac{n}{2}-1} \exp \left\{-\frac{n \beta}{2 \beta_{0}}\right\} .
$$

The $\chi^{2}$-distribution is a typical distribution that naturally arises in many circumstances, for example if $n$ independent Gaussian random variables $X_{i}, i=$ $1, \ldots, n$ with average 0 are squared and added. If we write

$$
\beta:=\sum_{i=1}^{n} X_{i}^{2}
$$

then $\beta$ has the probability density function (91). The average of the fluctuating $\beta$ is given by

$$
\langle\beta\rangle=n\left\langle X_{i}^{2}\right\rangle=\int_{0}^{\infty} \beta f(\beta) d \beta=\beta_{0}
$$

and the variance by

$$
\left\langle\beta^{2}\right\rangle-\beta_{0}^{2}=\frac{2}{n} \beta_{0}^{2} .
$$

The observed cosmic ray distribution at the earth does not contain any information on the local temperature at which the various particles were produced. Hence we have to average over all possible fluctuating temperatures, 
obtaining the measured energy spectrum as the marginal distribution

$$
p(E)=\int_{0}^{\infty} p(E \mid \beta) f(\beta) d \beta .
$$

The integral (13) with $f(\beta)$ given by (91) and $p(E \mid \beta)$ given by (17) is easily evaluated and one obtains

$$
p(E) \sim \frac{E^{2}}{(1+\tilde{\beta}(q-1) E)^{\frac{1}{q-1}}}
$$

with

$$
q=1+\frac{2}{n+6}
$$

and

$$
\tilde{\beta}=\frac{\beta_{0}}{4-3 q} .
$$

Eq. (14) is just the fitting function used in Fig. 1.

The variables $X_{i}$ describe the independent degrees of freedom contributing to the fluctuating temperature. At very large center of mass energies, the interaction region is very small, and all relevant degrees of freedom are basically represented by the 3 spatial dimensions into which heat can flow. We may physically interpret $X_{i}^{2}$ as the heat loss in the spatial $i$-direction, $i=x, y, z$, during the collision process that generates the cosmic ray particle. The more heat is lost, the smaller is the local $k T$, i.e. the larger is the local $\beta$ given by (10). The 3 spatial degrees of freedom yield $n=3$ or, according to (15),

$$
q=\frac{11}{9}=1.222 .
$$

For cosmic rays $E_{C M S}$ is very large, hence we expect a $q$-value that is close to this asymptotic value. The fit in Fig. 1 in fact uses $q=1.215$, which agrees with the predicted value in eq. (17) to about 3 digits. It also coincides well with the fitting value $q=1.225$ used by Tsallis et al. [14 using multiparameter generalizations of nonextensive canonical distributions.

For smaller center of mass energies, the interaction region will be bigger and more effective degrees of freedom within this bigger interaction region can contribute to the fluctuating temperature. Hence we expect that for finite $E_{C M S} n$ will be a bit larger than 3, or $q$ will be slightly smaller than 
11/9. A good parametrization, in agreement with experimentally measured cross sections in $e^{+} e^{-}$annihilation, is [7]

$$
q\left(E_{C M S}\right)=\frac{11-e^{-E_{C M S} / E_{0}}}{9+e^{-E_{C M S} / E_{0}}}
$$

with $E_{0} \approx 45.6 \mathrm{GeV}$.

Let us now predict a value for the other 'fitting parameter' entering into the nonextensive canonical distribution, the effective temperature $k \tilde{T} \approx 107$ $\mathrm{MeV}$. The Hagedorn temperature is experimentally measured to be about $k T_{H} \approx 180 \mathrm{MeV}$ [19, 20, which is the right order of magnitude. But why is the effective temperature fitting the cosmic ray spectrum smaller by a factor of about 0.6 as compared to the Hagedorn temperature?

A possible answer could be related to the way the Hagedorn temperature $T_{H}$ is experimentally determined. The experimental measurements are evaluated under the assumption that ordinary relativistic Maxwell-Boltzmann (MB) statistics applies, which is a good approximation for relatively small $E_{C M S}$. Assuming MB statistics

$$
p(E)=C E^{2} e^{-\beta E},
$$

the temperature $\beta^{-1}$ can be estimated from the maximum $E^{*}$ of the distribution as

$$
k T^{M B}=\frac{1}{2} E^{*} .
$$

This relation immediately follows from evaluating $p^{\prime}\left(E^{*}\right)=0$. However, suppose that in reality at sufficiently large $E_{C M S}$ Tsallis statistics

$$
p(E)=C \frac{E^{2}}{(1+(q-1) \tilde{\beta} E)^{\frac{1}{q-1}}}
$$

applies, due to the fluctuations mentioned before. Then the relation between the maximum and the temperature changes. One obtains from evaluating $p^{\prime}\left(E^{*}\right)=0$ the relation

$$
\tilde{\beta}^{-1}=k \tilde{T}=\frac{3-2 q}{2} E^{*}=(3-2 q) k T^{M B} .
$$

In collider experiments, the value of the maximum $E^{*}$ is seen to be essentially independent of $E_{C M S}$ [7]. An experimentally determined Hagedorn temperature (under the assumption of MB statistics) of $180 \pm 30 \mathrm{MeV}$ and a value of 
$q=1.215$ thus yields the effective temperature $k \tilde{T}=0.57 \cdot k T^{M B}=(103 \pm 17)$ $\mathrm{MeV}$. This just coincides (within the error bars) with the observed effective temperature that fits the data in Fig. 1. It also coincides with one of the two temperature fitting parameters $(96.15 \mathrm{MeV})$ used by Tsallis et al. in [14]. For $E_{C M S} \rightarrow \infty$ one obtains from eq. (17) and (22) the relation

$$
k \tilde{T}=\frac{5}{9} k T^{M B} .
$$

In this way all relevant parameters determining the shape of the cosmic ray energy spectrum are predicted from a simple theoretical consideration (except of course for the total flux rate of the cosmic rays, represented by the constant $C$ ).

To explain the 'knee' and 'ankle' in the cosmic ray spectrum we adopt the standard view [18. It is reasonable to assume that the knee at $E \approx 10^{16}$ $\mathrm{eV}$ is simply produced by the fact that one has reached the maximum energy scale to which typical galactic accelerators can accelerate. This naturally implies a rapid fall in the number of observed events with a higher energy, i.e. a steeper slope in Fig. 1 between about $10^{16}$ and $10^{19} \mathrm{eV}$. The 'ankle' at $E \approx 10^{19} \mathrm{eV}$ is then produced by the fact that a higher energy population of cosmic ray particles takes over from a lower energy population. This higher energy population may have a different origin (for example, extragalactic origin). The new population has a much smaller flux rate but can reach much larger energies. Naturally, the cosmic accelerators underlying the production process of this new species of cosmic rays must have a much larger center of mass energy $E_{C M S}$ than the ankle energy $\sim 10^{19} \mathrm{eV}$, so $q$ should be quite precisely given by its asymptotic value $11 / 9$, whereas the effective temperature $T$ should be the same as before. The dashed line in Fig. 1 corresponds to our formula with $q=11 / 9, k \tilde{T}=107 \mathrm{MeV}$ and a flux rate that is smaller by a factor $1 / 50$ as compared to the high-flux generation of cosmic rays. It is consistent with the data.

Our nonextensive theory predicts power-law behavior of the measured energy spectrum. For large $E$ one has $p(E) \sim E^{-\alpha}$ where the differential spectral index $\alpha$ is given by

$$
\alpha=\frac{1}{q-1}-2 .
$$

$q=1.215$ implies $\alpha=2.65$ (for moderately large energies), whereas for largest energies the asymptotic value $q=11 / 9$ implies $\alpha=5 / 2$. As shown 
in Fig. 2, the largest-energy events are compatible with such an asymptotic power law exponent, though of course the error bars of the experimental data are huge.

To conclude, our simple Hagedorn model based on ordinary statistical mechanics with local fluctuations of temperature very well reproduces the experimentally measured cosmic ray spectrum. By averaging over the temperature fluctuations one effectively ends up with a nonextensive theory, which describes the statistics of cosmic ray particles in a generalized statistical mechanics setting. Our model allows for concrete predictions of the relevant entropic index $q$ and of the relevant effective temperature parame-

ter $\tilde{T}$, and these predicted numerical values are in good agreement with the measured data.

\section{References}

[1] C. Tsallis, J. Stat. Phys. 52, 479 (1988)

[2] S. Abe, S. Martínez, F. Pennini, and A. Plastino, Phys. Lett. 281A, 126 (2001)

[3] C. Tsallis, R.S. Mendes and A.R. Plastino, Physica 261A, 534 (1998)

[4] C. Beck, G.S. Lewis and H.L. Swinney, Phys. Rev. 63E, 035303(R) (2001)

[5] C. Beck, K. Daniels and E. Bodenschatz, in preparation (2003)

[6] I. Bediaga, E.M.F. Curado, and J. Miranda, Physica 286A, 156 (2000)

[7] C. Beck, Physica 286A, 164 (2000)

[8] W.M. Alberico, A. Lavagno, P. Quarati, Eur. Phys. J. C 12, 499 (2000)

[9] A. Paramonov and A. Rostovtsev, hep-ph/0208233

[10] C. Beck, Spatio-temporal Chaos and Vacuum Fluctuations of Quantized Fields, World Scientific, Singapore (2002)

[11] G. Wilk and Z. Wlodarczyk, Phys. Rev. Lett. 84, 2770 (2000)

[12] C. Beck, Phys. Rev. Lett. 87, 180601 (2001)

[13] C. Beck, E.G.D. Cohen, Superstatistics, Physica A (in press) (2003)

[14] C. Tsallis, J.C. Anjos, E.P. Borges, astro-ph/0203258

[15] G. Kaniadakis, Phys. Rev. 66E, 056125 (2002) 
[16] R. Hagedorn, Nuovo Cimento 3, 147 (1965)

[17] http://astroparticle.uchicago.edu/announce.htm (data compilation by S.P. Swordy)

[18] T.K. Gaisser, T. Stanev, Cosmic Rays, in K. Hagiwara et al., Phys. Rev. D 66, 010001-1 (2002) (http://pdg.lbl.gov)

[19] W. Broniowski, W. Florkowski, Phys. Lett. 490B, 223 (2000) (hep-ph/0004104)

[20] F. Bucella, L. Popova, hep-ph/0209037

[21] D.J. Bird et al., Astrophys. J. 424, 491 (1994)

[22] M. Takeda et al., Phys. Rev. Lett. 81, 1163 (1998)

[23] M. Ave et al., astro-ph/0112253 


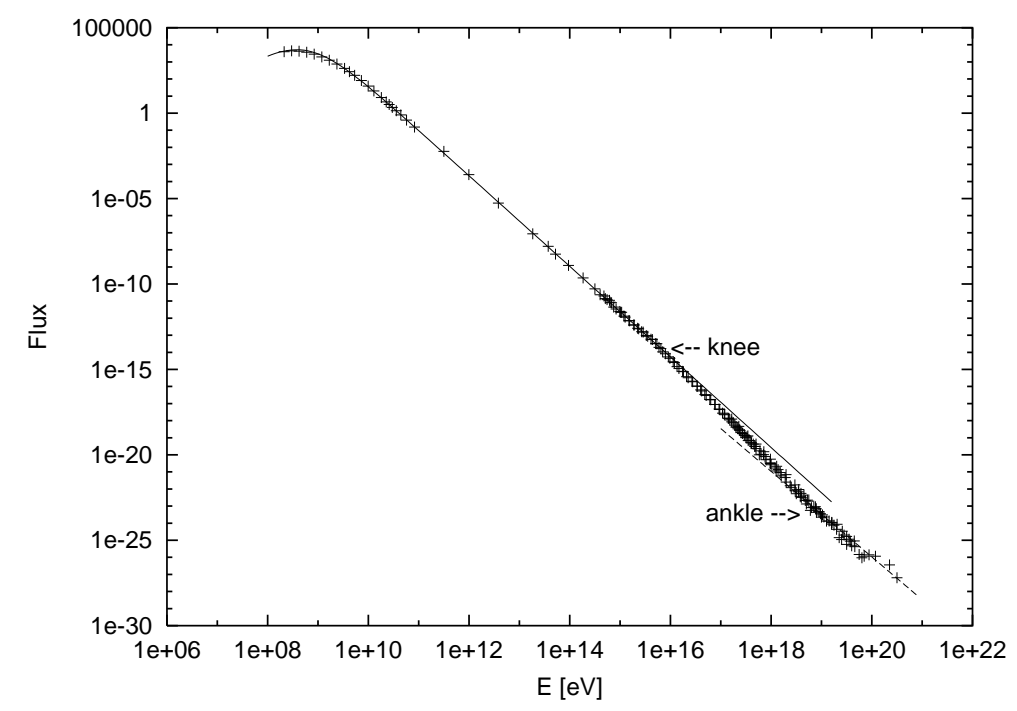

Fig. 1 Measured energy spectrum of primary cosmic rays (in units of $m^{-3} s^{-1} \mathrm{sr}^{-1} \mathrm{GeV}^{-1}$ ) as listed in [17. The solid line is the prediction (11) with $q=1.215, k \tilde{T}=107 \mathrm{MeV}$ and $C=5 \cdot 10^{-13}$ in the above units. The dashed line is eq. (11) with $q=11 / 9, k \tilde{T}=107 \mathrm{MeV}$ and $C$ smaller by a factor $1 / 50$. 


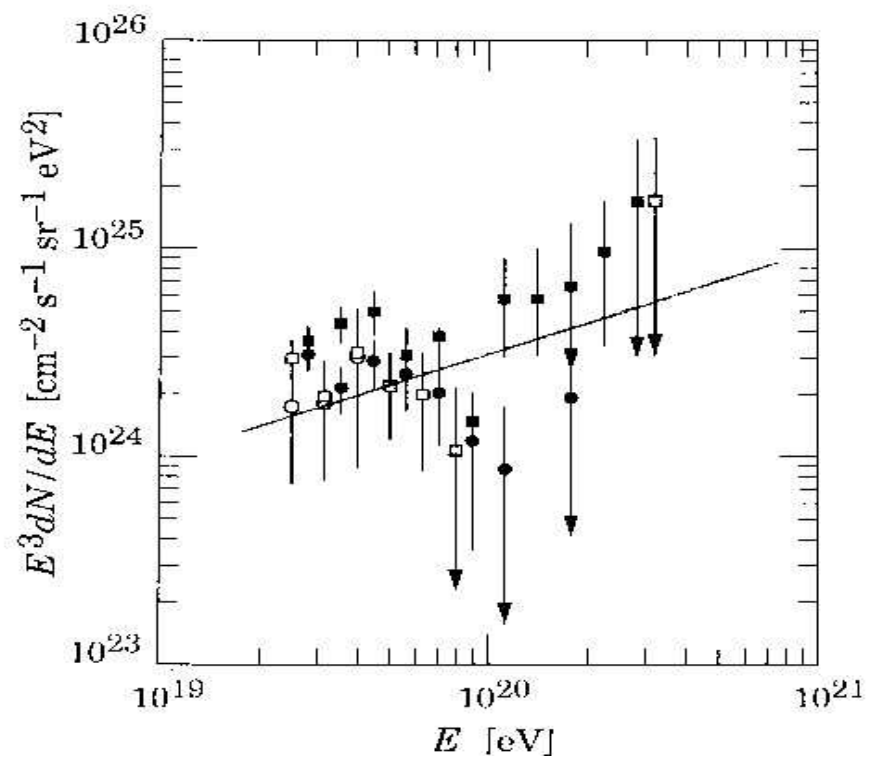

Fig. 2 Measured cosmic ray energy spectrum $E^{3} \cdot d N / d E$ at largest energies (data from [18, 21, 22, 23]). The straight line is the power law prediction with exponent $\alpha=5 / 2$ (corresponding to $q=11 / 9)$. 